Supplement of Adv. Geosci., 51, 1-14, 2019

https://doi.org/10.5194/adgeo-51-1-2019-supplement

(C) Author(s) 2019. This work is distributed under

the Creative Commons Attribution 4.0 License.

(c) (1)

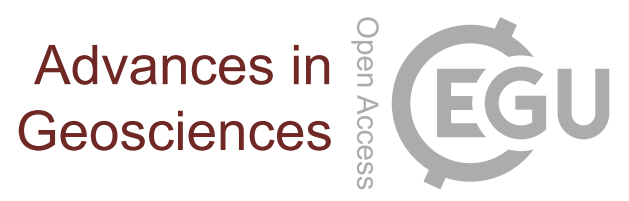

Supplement of

\title{
2016 Central Italy Earthquakes: comparison between GPS signals and low-cost distributed MEMS arrays
}

Nicola Cenni et al.

Correspondence to: Jacopo Boaga (jacopo.boaga@unipd.it)

The copyright of individual parts of the supplement might differ from the CC BY 4.0 License. 
table1a

\begin{tabular}{|c|c|c|c|c|c|}
\hline Id Code & Lon. & Lat & North (mm) & East (mm) & Vertical (mm) \\
\hline AQUI & 13.35 & 42.37 & $-1.6 \pm 0.9$ & $-0.6 \pm 0.6$ & $-0.3 \pm 1.8$ \\
\hline INGR & 12.51 & 41.83 & $-0.3 \pm 0.6$ & $-0.0 \pm 0.6$ & $0.7 \pm 1.5$ \\
\hline SIEN & 11.34 & 43.31 & $-0.8 \pm 0.6$ & $0.5 \pm 0.6$ & $3.7 \pm 1.8$ \\
\hline UNPG & 12.36 & 43.12 & $-0.5 \pm 0.6$ & $-0.5 \pm 0.6$ & $-1.2 \pm 1.8$ \\
\hline MAON & 11.13 & 42.43 & $-0.1 \pm 0.9$ & $0.1 \pm 0.6$ & $-4.0 \pm 2.7$ \\
\hline RSTO & 14 & 42.66 & $0.4 \pm 0.6$ & $2.7 \pm 0.6$ & $-0.5 \pm 1.5$ \\
\hline TOLF & 12 & 42.06 & $0.2 \pm 0.6$ & $1.0 \pm 0.9$ & $0.3 \pm 1.5$ \\
\hline FRES & 14.67 & 41.97 & $-0.0 \pm 1.2$ & $0.2 \pm 0.9$ & $3.2 \pm 3.6$ \\
\hline UNTR & 12.67 & 42.56 & $-3.1 \pm 0.9$ & $-6.5 \pm 0.9$ & $-0.5 \pm 2.1$ \\
\hline MOSE & 12.49 & 41.89 & $-1.3 \pm 0.6$ & $-0.3 \pm 0.6$ & $-3.2 \pm 1.5$ \\
\hline REPI & 12 & 42.95 & $0.6 \pm 0.9$ & $-1.1 \pm 0.9$ & $-3.4 \pm 2.4$ \\
\hline ITGT & 12.78 & 43.23 & $-0.1 \pm 1.2$ & $-0.4 \pm 1.5$ & $-3.7 \pm 3.6$ \\
\hline ALRA & 14.03 & 41.73 & $-0.5 \pm 1.2$ & $0.9 \pm 1.5$ & $-2.8 \pm 3.3$ \\
\hline AQRA & 13.37 & 42.37 & $-0.4 \pm 0.9$ & $-1.0 \pm 0.9$ & $1.9 \pm 2.4$ \\
\hline BLRA & 13.56 & 41.81 & $1.2 \pm 1.2$ & $1.8 \pm 1.5$ & $2.0 \pm 3.3$ \\
\hline CDRA & 13.72 & 42.37 & $0.2 \pm 0.9$ & $0.1 \pm 0.9$ & $3.2 \pm 2.7$ \\
\hline FRRA & 14.29 & 42.42 & $-0.8 \pm 0.6$ & $0.1 \pm 0.6$ & $2.0 \pm 1.8$ \\
\hline MRRA & 13.92 & 42.89 & $0.9 \pm 0.6$ & $3.7 \pm 0.6$ & $-0.1 \pm 1.8$ \\
\hline MTRA & 13.24 & 42.53 & $-10.1 \pm 0.9$ & $-7.9 \pm 0.6$ & $-0.3 \pm 2.7$ \\
\hline OCRA & 13.04 & 42.05 & $0.6 \pm 0.9$ & $-0.3 \pm 0.9$ & $2.2 \pm 2.1$ \\
\hline OVRA & 13.52 & 42.14 & $0.8 \pm 1.8$ & $-1.4 \pm 1.5$ & $-0.4 \pm 3.9$ \\
\hline SCRA & 14 & 42.27 & $-1.3 \pm 0.6$ & $0.2 \pm 0.9$ & $2.3 \pm 2.7$ \\
\hline SMRA & 13.92 & 42.05 & $-1.0 \pm 0.9$ & $-1.4 \pm 0.6$ & $-1.0 \pm 3.0$ \\
\hline TERA & 13.7 & 42.66 & $-0.8 \pm 0.6$ & $8.3 \pm 0.6$ & $2.5 \pm 2.1$ \\
\hline VTRA & 14.71 & 42.11 & $-0.6 \pm 0.6$ & $0.3 \pm 0.6$ & $-0.5 \pm 1.8$ \\
\hline OTRA & 13.65 & 41.95 & $0.1 \pm 1.5$ & $-3.7 \pm 1.2$ & $-0.9 \pm 3.9$ \\
\hline ALAT & 13.38 & 41.67 & $1.2 \pm 0.9$ & $-0.8 \pm 0.6$ & $1.7 \pm 2.1$ \\
\hline RIET & 12.86 & 42.41 & $-4.3 \pm 0.6$ & $-6.4 \pm 0.6$ & $-0.8 \pm 2.1$ \\
\hline OLGI & 12.36 & 42.05 & $-2.3 \pm 0.6$ & $-1.9 \pm 0.6$ & $-1.8 \pm 1.8$ \\
\hline VIT1 & 12.1 & 42.43 & $-0.4 \pm 0.6$ & $-2.9 \pm 0.6$ & $-1.6 \pm 1.8$ \\
\hline TARQ & 11.76 & 42.25 & $-0.1 \pm 0.6$ & $-1.6 \pm 0.6$ & $-2.2 \pm 1.8$ \\
\hline PAGL & 14.5 & 42.16 & $-0.3 \pm 0.6$ & $-0.4 \pm 0.6$ & $0.2 \pm 2.4$ \\
\hline PSAN & 14.14 & 42.52 & $-0.4 \pm 0.6$ & $-1.5 \pm 0.6$ & $1.5 \pm 1.8$ \\
\hline GRAM & 13.87 & 42.98 & $2.4 \pm 0.6$ & $5.0 \pm 0.6$ & $0.1 \pm 1.5$ \\
\hline ASCC & 13.59 & 42.86 & $6.8 \pm 0.6$ & $15.9 \pm 0.6$ & $-0.9 \pm 2.4$ \\
\hline ANCG & 13.5 & 43.6 & $-0.0 \pm 0.6$ & $1.9 \pm 0.3$ & $-2.7 \pm 1.5$ \\
\hline MACE & 13.45 & 43.29 & $2.6 \pm 0.9$ & $-0.7 \pm 0.6$ & $1.8 \pm 2.7$ \\
\hline FOSS & 12.81 & 43.69 & $-0.1 \pm 0.6$ & $-1.1 \pm 0.6$ & $-0.1 \pm 1.8$ \\
\hline TERI & 12.65 & 42.57 & $-3.0 \pm 0.6$ & $-4.6 \pm 0.6$ & $-1.4 \pm 2.1$ \\
\hline GUB2 & 12.58 & 43.35 & $0.5 \pm 0.9$ & $0.1 \pm 0.9$ & $-2.1 \pm 3.3$ \\
\hline FRMO & 13.73 & 43.17 & $0.7 \pm 0.6$ & $2.6 \pm 0.6$ & $-1.5 \pm 2.1$ \\
\hline FOL1 & 12.7 & 42.95 & $-0.6 \pm 0.6$ & $-3.4 \pm 0.6$ & $-2.1 \pm 2.4$ \\
\hline CIT1 & 12.25 & 43.47 & $0.8 \pm 0.9$ & $0.5 \pm 0.6$ & $-3.8 \pm 2.7$ \\
\hline CAMU & 11.98 & 43.26 & $-0.4 \pm 0.9$ & $-0.8 \pm 0.6$ & $2.6 \pm 2.4$ \\
\hline
\end{tabular}


table1a

\begin{tabular}{|c|c|c|c|c|c|}
\hline PITI & 11.67 & 42.63 & $-0.3 \pm 0.9$ & $-1.6 \pm 0.9$ & $-2.4 \pm 2.7$ \\
\hline MCIN & 11.49 & 43.06 & $-0.2 \pm 0.6$ & $-0.9 \pm 0.6$ & $2.4 \pm 2.4$ \\
\hline GAVO & 10.89 & 42.94 & $-0.4 \pm 0.9$ & $-0.3 \pm 0.6$ & $-2.5 \pm 2.4$ \\
\hline $\mathrm{PIBI}$ & 12.45 & 43.13 & $-0.2 \pm 0.6$ & $0.3 \pm 0.9$ & $-2.3 \pm 3.0$ \\
\hline PSTE & 11.12 & 42.43 & $0.6 \pm 1.2$ & $-1.0 \pm 0.6$ & $14.7 \pm 3.9$ \\
\hline TERN & 12.63 & 42.57 & $-3.2 \pm 0.6$ & $-5.6 \pm 0.6$ & $-1.2 \pm 2.4$ \\
\hline ROAN & 12.42 & 41.9 & $-1.2 \pm 0.6$ & $-0.6 \pm 0.6$ & $-0.4 \pm 1.8$ \\
\hline CAMG & 13.07 & 43.14 & $-0.0 \pm 0.9$ & $0.7 \pm 0.9$ & $0.8 \pm 2.1$ \\
\hline FIOR & 11.59 & 42.83 & $0.8 \pm 0.9$ & $-1.8 \pm 0.9$ & $-0.6 \pm 2.4$ \\
\hline GEOT & 13.51 & 43.57 & $0.5 \pm 0.6$ & $0.0 \pm 0.6$ & $-1.7 \pm 2.4$ \\
\hline GINE & 13.38 & 43.12 & $3.4 \pm 0.6$ & $0.9 \pm 0.6$ & $-2.1 \pm 1.8$ \\
\hline GRTG & 13.87 & 42.97 & $1.7 \pm 0.6$ & $4.9 \pm 0.6$ & $0.5 \pm 2.1$ \\
\hline MAGL & 13.59 & 43.14 & $3.7 \pm 0.6$ & $2.4 \pm 0.6$ & $-1.3 \pm 1.8$ \\
\hline MOIE & 13.12 & 43.5 & $0.0 \pm 0.6$ & $0.7 \pm 0.6$ & $1.1 \pm 1.8$ \\
\hline PSST & 11.12 & 42.43 & $-0.5 \pm 0.9$ & $-1.6 \pm 0.6$ & $0.5 \pm 2.4$ \\
\hline PULC & 11.82 & 43.16 & $-0.1 \pm 0.9$ & $-1.5 \pm 0.9$ & $0.5 \pm 2.7$ \\
\hline SENI & 13.21 & 43.71 & $0.2 \pm 0.6$ & $-0.0 \pm 0.6$ & $0.4 \pm 1.8$ \\
\hline AVZZ & 13.45 & 42.03 & $-2.5 \pm 0.9$ & $-0.8 \pm 0.9$ & $0.9 \pm 2.4$ \\
\hline CIST & 12.83 & 41.58 & $1.0 \pm 0.9$ & $1.4 \pm 0.9$ & $-4.5 \pm 2.7$ \\
\hline FIAN & 12.59 & 42.16 & $-2.7 \pm 0.9$ & $-2.1 \pm 0.6$ & $0.1 \pm 2.4$ \\
\hline ITRA & 14 & 42.66 & $-0.3 \pm 0.6$ & $3.4 \pm 0.6$ & $3.6 \pm 1.8$ \\
\hline LANC & 14.38 & 42.2 & $-0.3 \pm 0.6$ & $-0.1 \pm 0.6$ & $2.5 \pm 2.1$ \\
\hline PAGA & 13.47 & 42.36 & $0.2 \pm 0.9$ & $-1.2 \pm 0.6$ & $0.6 \pm 2.4$ \\
\hline PESC & 14.2 & 42.47 & $-0.6 \pm 0.9$ & $0.5 \pm 0.9$ & $1.2 \pm 2.7$ \\
\hline VITE & 12.11 & 42.42 & $-1.1 \pm 0.6$ & $-1.9 \pm 0.6$ & $-3.0 \pm 1.8$ \\
\hline ZAGA & 12.75 & 41.86 & $-1.8 \pm 0.9$ & $1.8 \pm 0.9$ & $-3.2 \pm 2.4$ \\
\hline ATBU & 12.55 & 43.48 & $-8.8 \pm 1.2$ & $-11.7 \pm 1.2$ & $18.2 \pm 3.0$ \\
\hline ATFO & 12.57 & 43.37 & $-0.3 \pm 0.9$ & $-1.4 \pm 0.9$ & $1.4 \pm 2.1$ \\
\hline ATLO & 12.41 & 43.32 & $-1.2 \pm 0.6$ & $1.0 \pm 0.6$ & $1.1 \pm 1.8$ \\
\hline ATMI & 12.27 & 43.33 & $0.4 \pm 0.6$ & $0.7 \pm 0.6$ & $0.4 \pm 1.8$ \\
\hline ATTE & 12.35 & 43.2 & $-0.4 \pm 0.6$ & $-0.3 \pm 0.6$ & $1.8 \pm 1.5$ \\
\hline CAFI & 11.97 & 43.33 & $-1.2 \pm 0.9$ & $-0.1 \pm 0.6$ & $4.3 \pm 2.1$ \\
\hline CESI & 12.9 & 43.01 & $-0.4 \pm 0.6$ & $-1.5 \pm 0.6$ & $2.7 \pm 1.8$ \\
\hline CSSB & 12.25 & 43.21 & $-0.4 \pm 0.6$ & $-0.9 \pm 0.6$ & $-0.4 \pm 1.5$ \\
\hline MGAB & 12.11 & 42.91 & $0.1 \pm 0.6$ & $-2.0 \pm 0.6$ & $-0.0 \pm 1.8$ \\
\hline MUR1 & 12.52 & 43.26 & $-0.8 \pm 0.6$ & $0.8 \pm 0.9$ & $1.2 \pm 1.8$ \\
\hline MVAL & 12.41 & 43.38 & $-0.5 \pm 0.6$ & $0.5 \pm 0.6$ & $-0.3 \pm 1.8$ \\
\hline PIET & 12.4 & 43.45 & $-0.4 \pm 0.6$ & $0.8 \pm 0.6$ & $1.9 \pm 1.5$ \\
\hline SACS & 11.91 & 42.85 & $-0.2 \pm 0.9$ & $-1.9 \pm 0.9$ & $-0.7 \pm 2.1$ \\
\hline VALC & 12.28 & 43.28 & $-0.4 \pm 0.6$ & $-0.6 \pm 0.6$ & $-0.4 \pm 1.5$ \\
\hline UMBE & 12.33 & 43.31 & $0.4 \pm 0.6$ & $0.6 \pm 0.6$ & $5.2 \pm 2.1$ \\
\hline ANCN & 13.53 & 43.61 & $5.2 \pm 1.2$ & $0.7 \pm 0.6$ & $1.4 \pm 1.8$ \\
\hline GUMA & 13.34 & 43.06 & $0.6 \pm 0.9$ & $-0.2 \pm 0.9$ & $-3.9 \pm 2.7$ \\
\hline MLAG & 12.78 & 43.43 & $-1.9 \pm 0.9$ & $-1.8 \pm 0.9$ & $-0.2 \pm 1.8$ \\
\hline GUAR & 13.31 & 41.79 & $-1.3 \pm 0.6$ & $-0.5 \pm 0.6$ & $0.7 \pm 2.4$ \\
\hline
\end{tabular}


table1a

\begin{tabular}{|c|c|c|c|c|c|}
\hline MTER & 13.21 & 42.51 & $-9.8 \pm 0.9$ & $-5.0 \pm 0.6$ & $1.9 \pm 2.1$ \\
\hline BARS & 13.58 & 42.34 & $-1.0 \pm 0.9$ & $-0.3 \pm 0.6$ & $2.0 \pm 2.4$ \\
\hline CAOC & 13.48 & 42.29 & $-2.8 \pm 1.5$ & $-3.0 \pm 0.9$ & $2.2 \pm 2.1$ \\
\hline CERT & 12.98 & 41.95 & $-1.5 \pm 0.6$ & $-0.9 \pm 0.6$ & $1.3 \pm 1.8$ \\
\hline CONI & 13.39 & 42.41 & $-0.3 \pm 0.9$ & $-2.0 \pm 0.6$ & $3.9 \pm 2.1$ \\
\hline GNAL & 13.52 & 42.58 & $-1.4 \pm 0.9$ & $4.9 \pm 0.9$ & $-0.7 \pm 2.1$ \\
\hline LNSS & 13.04 & 42.6 & $-16.0 \pm 0.6$ & $-20.7 \pm 0.9$ & $4.5 \pm 2.4$ \\
\hline LPEL & 14.18 & 42.05 & $-1.1 \pm 0.9$ & $0.4 \pm 1.2$ & $1.2 \pm 3.3$ \\
\hline MTTO & 12.99 & 42.46 & $-7.9 \pm 0.6$ & $-7.8 \pm 0.9$ & $2.7 \pm 2.4$ \\
\hline POFI & 13.71 & 41.72 & $0.9 \pm 0.6$ & $-1.4 \pm 0.6$ & $1.2 \pm 1.8$ \\
\hline RDPI & 12.71 & 41.76 & $0.3 \pm 0.6$ & $-0.7 \pm 0.6$ & $-2.3 \pm 1.8$ \\
\hline RMPO & 12.7 & 41.81 & $-0.4 \pm 0.6$ & $-1.1 \pm 0.6$ & $-0.9 \pm 2.1$ \\
\hline RNI2 & 14.15 & 41.7 & $-0.1 \pm 0.6$ & $-0.4 \pm 0.6$ & $-0.4 \pm 1.8$ \\
\hline ROPI & 13.34 & 42.33 & $-1.0 \pm 0.6$ & $-2.7 \pm 0.6$ & $0.0 \pm 1.8$ \\
\hline SGRE & 13.5 & 42.34 & $-1.7 \pm 0.6$ & $-2.2 \pm 0.6$ & $3.5 \pm 2.1$ \\
\hline TRIV & 14.55 & 41.77 & $0.1 \pm 0.6$ & $0.0 \pm 0.6$ & $0.7 \pm 2.1$ \\
\hline VVLO & 13.62 & 41.87 & $1.2 \pm 0.6$ & $0.1 \pm 0.6$ & $-4.3 \pm 1.8$ \\
\hline CAMR & 13.07 & 43.14 & $1.6 \pm 0.9$ & $-1.0 \pm 0.6$ & $0.3 \pm 2.4$ \\
\hline SULM & 13.93 & 42.04 & $-1.3 \pm 0.9$ & $0.2 \pm 0.9$ & $-4.9 \pm 3.3$ \\
\hline ACQL & 11.86 & 42.74 & $-2.9 \pm 0.9$ & $-0.9 \pm 0.9$ & $-2.5 \pm 2.1$ \\
\hline AMAT & 13.29 & 42.63 & $23.2 \pm 0.9$ & $-7.7 \pm 0.6$ & $-50.7 \pm 2.4$ \\
\hline ARDE & 12.54 & 41.61 & $-0.5 \pm 0.6$ & $-0.5 \pm 0.6$ & $-0.7 \pm 1.8$ \\
\hline CVTV & 11.8 & 42.1 & $-1.2 \pm 0.6$ & $-1.2 \pm 0.6$ & $-3.3 \pm 1.8$ \\
\hline FIUM & 12.23 & 41.77 & $-0.7 \pm 0.6$ & $-0.6 \pm 0.6$ & $-1.2 \pm 1.8$ \\
\hline FROS & 13.35 & 41.64 & $1.2 \pm 0.9$ & $-1.1 \pm 0.6$ & $-3.7 \pm 2.1$ \\
\hline MOCL & 11.6 & 42.35 & $-0.6 \pm 0.6$ & $-1.2 \pm 0.6$ & $-2.8 \pm 1.5$ \\
\hline RIFL & 12.48 & 42.21 & $-2.0 \pm 0.6$ & $-1.9 \pm 0.6$ & $-0.2 \pm 2.1$ \\
\hline ROUN & 12.49 & 41.89 & $-0.7 \pm 0.6$ & $-0.4 \pm 0.6$ & $-1.2 \pm 1.8$ \\
\hline RITI & 12.86 & 42.41 & $-17.1 \pm 2.1$ & $-22.9 \pm 1.5$ & $9.1 \pm 5.4$ \\
\hline VALM & 12.92 & 41.78 & $-0.5 \pm 0.9$ & $-0.1 \pm 0.6$ & $-0.5 \pm 2.1$ \\
\hline VIRB & 12.11 & 42.42 & $-1.1 \pm 0.6$ & $-1.1 \pm 0.6$ & $-2.6 \pm 1.8$ \\
\hline VIVA & 12.89 & 42.02 & $-2.0 \pm 0.6$ & $-0.3 \pm 0.6$ & $0.2 \pm 2.4$ \\
\hline
\end{tabular}


table1a

Pagina 4 